\title{
To delay or not frozen embryo transfer in freeze-all cycles?
}

\author{
Panagiotis Drakopoulos $^{1,2}$, Samuel Santos-Ribeiro ${ }^{3}$, Shari Mackens ${ }^{1}$, Anna Lisa Racca ${ }^{1}$, Christophe \\ Blockeel $^{1,4}$, Herman Tournaye ${ }^{1}$, Antonis Makrigiannakis ${ }^{2}$
}

${ }^{1}$ Centre for Reproductive Medicine, Universitair Ziekenhuis Brussel, Vrije Universiteit Brussel, Brussels, Belgium; ${ }^{2}$ Department of Obstetrics and Gynecology, University General Hospital of Heraklion, Medical School, University of Crete, Heraklion, Greece; ${ }^{3}$ IVI-RMA Lisboa, Lisbon, Portugal; ${ }^{4}$ Department of Obstetrics and Gynecology, University of Zagreb-School of Medicine, Zagreb, Croatia

Correspondence to: Panagiotis Drakopoulos, MD, PhD. Centre for Reproductive Medicine, Universitair Ziekenhuis Brussel, Vrije Universiteit Brussel, Laarbeeklaan 101 - 1090 Brussel, Belgium. Email: panagiotisdrakopoulos@hotmail.com.

Provenance and peer review: This article was commissioned by the editorial office, Annals of Translational Medicine. The article did not undergo external peer review.

Comment on: Huang J, Lu X, Xie Q, et al. Timing of frozen-thawed embryo transfer after controlled ovarian stimulation in a non-elective freeze-all policy. Ann Transl Med 2019;7:752.

Submitted Feb 29, 2020. Accepted for publication Mar 20, 2020.

doi: 10.21037/atm.2020.03.209

View this article at: http://dx.doi.org/10.21037/atm.2020.03.209

\section{Frozen versus fresh cycles in IVF}

The practice of freezing embryos for deferred frozen embryo transfer (FET) has given rise to the so-called freezeall strategy. The freeze-all policy was first introduced by clinicians in an attempt to prevent ovarian hyperstimulation syndrome (OHSS) (1) and has increasingly been integrated into in vitro fertilization (IVF) for several indications, including among others progesterone rise at the end of the follicular phase, pre-implantation genetic testing for aneuploidy and embryo pooling in patients with low ovarian reserve (2). Freeze-all may be planned and then is referred to as "elective" freeze-all, while in some cases unplanned (or "non-elective") freezing is performed. Given the popularity of the freeze-all protocol and the dramatic increase of FET cycles over the last decade, several RCTs and meta-analyses aimed to evaluate the efficacy of FET versus fresh ET in different IVF populations.

In particular, Bosdou et al. (3) conducted a meta-analysis that consisted of 4 RCTs ( $n=3,255$ patients), comparing the first FET (in a freeze-all strategy) to a fresh embryo transfer (ET) in normal responders and 4 RCTs ( $\mathrm{n}=2,010$ patients) in high responders. In high responders, a significantly higher probability of live birth was observed in the FET group, while in normal responders no significant difference in live birth rates (LBR) between FET and fresh ET was detected. These findings were similar to those reported by Roque et al. (4) who reported a benefit with FET solely in hyper-responders, after evaluation of 11 studies, including 5,379 patients.

Two recent large RCTs offered new insights into the efficacy of the freeze-all strategy. Both studies were practically identical in design in that they tested freezeall cycle outcomes with single ET in comparison to fresh transfer. The only difference was that in one study ET took place at the blastocyst stage (5) and in the other at the cleavage stage (6). Patients with blastocyst transfer undergoing FET were found to have improved outcomes compared to fresh ET counterparts, while those with cleavage stage transfer did not. A possible explanation for this discrepancy may be that patients with blastocyst-stage ET are favorably selected (i.e., similar to high-responders), whereas patients with cleavage-stage ET are much less favorably selected.

To summarize, FET in a freeze-all context may have an advantage over fresh ET in good prognosis women, but not in average and certainly not in poor prognosis patients.

\section{When should FET take place?}

Physicians are frequently asked whether a recent ovarian stimulation attempt may pose any problem to a subsequent FET, especially following GnRH agonist triggering given the abrupt luteolysis which may perceived as harmful. However, despite the ideal timing of a subsequent FET 
Table 1 Observational studies evaluating the effect of FET timing on pregnancy outcomes after the freeze-all strategy

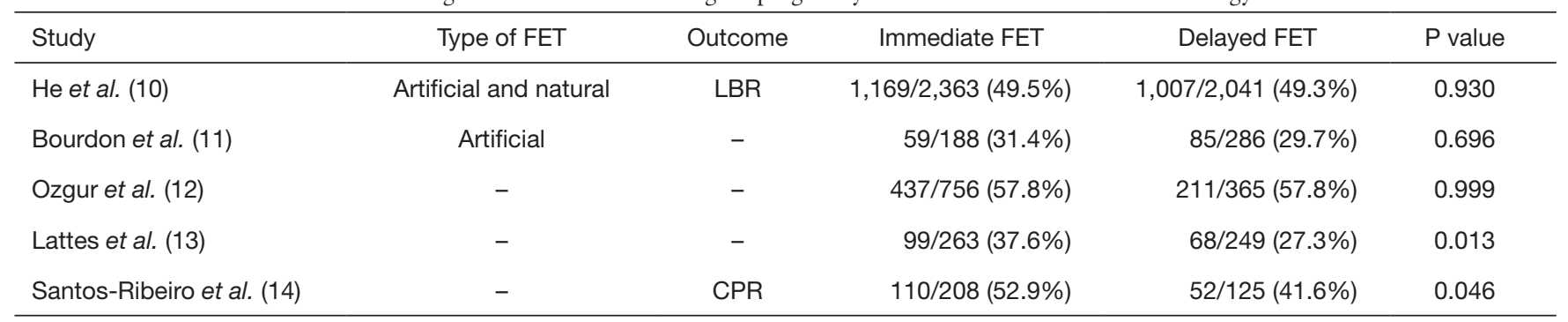

LBR, live birth rates; CPR, clinical pregnancy rates.

being a common concern, this had been a relatively unstudied topic until 2016. Therefore, even with the lack of evidence, it was rather common place to recommend waiting at least one menstrual cycle before the first FET after a freeze-all cycle, a habit which may have mirrored the empirical decision taken by the first researchers performing RCTs to assess the efficacy of the freeze-all strategy (7-9). With this waiting period, clinicians intended to circumvent any potential hindering effect that the abrupt luteolysis following GnRH agonist triggering could have on the endometrium in a subsequent cycle. While covered by the best of intentions, the lack of clear scientific evidence to support this practice may have caused unnecessary distress to infertile patients eager to conceive as soon as possible. Hence, an increasing number of centers also started to offer the option to perform FETs in the first menstrual cycle immediately after GnRH agonist triggering. Ever since, a series of studies with either discrepant or difficult to explain results have followed, leaving the challenging question of the ideal timing for FET still open for debate.

In this regard, evidence regarding the ideal FET timing (immediate versus delayed) after the freeze-all strategy mainly derives from studies, which included only artificial FET cycles (Table 1). Santos-Ribeiro et al. (14) were the first to compare both FET timing by combining data of 333 FETs from two centers. In this study, the researchers noted a difficult to explain trend towards improved clinical pregnancy rates $(\mathrm{CPR})$ in immediate FETs, which disappeared following adjustment for potential confounding. Shortly after, three additional studies complemented these data by reporting on live birth rates (LBR) as the main outcome (11-13). While overall these studies failed to show any difference between the two FET timings, Lattes et al. (13) also hinted towards a potential improvement in LBR after immediate FET that no longer remained significant following adjustment for potential confounding. Last year, an RCT attempting to validate prospectively these findings had its first results presented at the ESHRE annual meeting (15). The updated results presented during the conference seemed to confirm that immediate FET improved ongoing pregnancy rates $(47.2 \%$ vs. $39.3 \%, \mathrm{P}=0.03)$ and reduced the risk of miscarriage (11.2 vs. $19.7 \%, \mathrm{P}=0.02)$. However, this study was limited not only by the fact that it included both patients with a previous failed fresh embryo transfer and those who performed freeze-all, but most importantly by the unequal balance of the subjects in terms of female age and number of oocytes retrieved, despite randomization. Hence, until this issue is clarified further in future studies, it seems that we are still unable to determine which timing after freeze-all is the best for an artificial FET cycle.

On the other hand, a few studies have recently also attempted to evaluate whether the timing had any influence in natural cycle FETs. Higgins et al. (16) reviewed 4994 natural and artificial FETs and concluded that immediate FET was associated with improved LBR. Meanwhile, Kaye et al. (17) analysed 344 blastocyst FETs and found a trend towards a potential benefit in delaying a cycle before proceeding with FET. Besides the aforementioned discrepancy, these results are also difficult to extrapolate directly to the freeze-all setting since they also included FETs performed after a failed fresh embryo transfer in the sample. Hence, studies including natural cycle FETs after freeze-all, such as the those published recently by Huang et al. (18) and He et al. (10), are of added value for clinicians to assess whether immediate natural FET cycles may be safe to perform or not.

\section{Commentary on the current study}

The retrospective study of Huang et al. (18) included 2998 
patients who underwent their first FET using an artificial or modified natural protocol, following conventional ovarian stimulation (COS) in a progesterone primed (PPOS) or gonadotropin-releasing hormone agonist (GnRH-a) short protocol and freeze-all. Therefore, the strategy of freezeall was mandatory for several patients (PPOS group) and should be classified as an elective one (in contrast to authors' statement). The population was divided into the "immediate" FET and "delayed FET" group (average 91 days after COS). The primary outcome of the study was LBR, which were found to be significantly higher in the immediate group compared to the delayed group (55.7\% vs. $43.8 \%, \mathrm{P}=0.002)$, leading to the conclusion that FET should be performed at the earliest after COS, in case of freeze-all. The major strength of the study relies on the rigorous methodological approach (propensity score matching) and the large sample size. Nonetheless, the retrospective nature of the study is inherent to risk of bias. Selection bias may have occurred given that the reasons for delayed FET were not reported and several patients may have been advised by their physician to delay FET in case of comorbidities. Furthermore, policies like double cleavage stage ET (which was performed in approximately $90 \%$ of cases) are not relevant in modern IVF practice $(19,20)$. Thus, the aforementioned limitations preclude generalizability of the results and future prospective studies are required.

\section{Why would an immediate FET perform better?}

So far, no head-to-head comparison of the endometrial receptivity profile between immediate and delayed FET has been performed and most studies have shown no difference in reproductive outcomes (Table 1). The findings of Huang et al. (18) give new insights into the complex procedure of implantation and food for thought for the conduction of further basic and clinical research on the topic. Several assumptions can be made for the better outcomes in case of immediate FET. One hypothesis would be that the abrupt luteolysis following $\mathrm{GnRH}$ agonist triggering may cause a sharp decrease in circulating progesterone levels that could be beneficial, given that previous studies have shown that elevated progesterone levels in the beginning of COS are associated with a decreased chance of pregnancy (21). Other mechanisms may include a more favourable endometrial immune status after COS (22), while the corpora lutea producing high levels of vasodilatory and angiogenic factors during stimulation (23) could also have a positive effect in case of an immediate FET. Lastly, endometrial gene expression studies have shown an altered endometrial profile in COS cycles (24) and it is currently unknown whether there is a carry-over effect to the next(s) cycle(s). If we further take into consideration the context of FET (previous failed fresh embryo transfer or freeze-all), the various indications of freeze-all (elective $v s$. non elective), the stage of ET, number of embryos transferred and different FET preparation protocols, it becomes clear that more evidence is warranted before drawing firm conclusions.

\section{Conclusions}

In conclusion, based on the available evidence, reproductive outcomes following immediate FET do not seem to be inferior to delayed FET in freeze-all cycles. The findings by Huang et al. are intriguing and should be certainly taken into consideration. Nonetheless, the observational nature of existing studies, along with their limitations, makes room only for speculations. Further prospective clinical and translational studies are necessary in order to validate these findings, as well as to investigate the underlying mechanisms.

\section{Acknowledgments}

Funding: None.

\section{Footnote}

Conflicts of Interest: All authors have completed the ICMJE uniform disclosure form (available at http://dx.doi. org/10.21037/atm.2020.03.209). HT reports grants from Merck, grants from MSD, grants from Goodlife, grants from Cook, grants from Roche, grants from Besins, other from Finox, other from Abbott, other from ObsEva, grants from Ferring, grants from Research Fund of Flanders (FWO), other from Ovascience, grants from Mithra (now Allergan), outside the submitted work. SSR reports and has received lecturing fees from M.S.D., Ferring and Besins, and research grants from M.S.D. CB reports honoraria and/ or research grants from MSD, Ferring, Gedeon Richter, Abbott. PD reports grants and personal fees from MSD, personal fees from Ferring, personal fees from Merck Serono, outside the submitted work. The other authors have no conflicts of interest to declare.

Ethical Statement: The authors are accountable for all 
aspects of the work in ensuring that questions related to the accuracy or integrity of any part of the work are appropriately investigated and resolved.

Open Access Statement: This is an Open Access article distributed in accordance with the Creative Commons Attribution-NonCommercial-NoDerivs 4.0 International License (CC BY-NC-ND 4.0), which permits the noncommercial replication and distribution of the article with the strict proviso that no changes or edits are made and the original work is properly cited (including links to both the formal publication through the relevant DOI and the license). See: https://creativecommons.org/licenses/by-nc-nd/4.0/.

\section{References}

1. Devroey P, Polyzos NP, Blockeel C. An OHSS-Free Clinic by segmentation of IVF treatment. Hum Reprod 2011;26:2593-7.

2. Blockeel C, Drakopoulos P, Santos-Ribeiro S, et al. A fresh look at the freeze-all protocol: a SWOT analysis. Hum Reprod 2016;31:491-7.

3. Bosdou JK, Venetis CA, Tarlatzis BC, et al. Higher probability of live-birth in high, but not normal, responders after first frozen-embryo transfer in a freezeonly cycle strategy compared to fresh-embryo transfer: a meta-analysis. Hum Reprod 2019;34:491-505.

4. Roque M, Haahr T, Geber S, et al. Fresh versus elective frozen embryo transfer in IVF/ICSI cycles: a systematic review and meta-analysis of reproductive outcomes. Hum Reprod Update 2019;25:2-14.

5. Wei D, Zhang H, Legro RS, et al. Fresh versus frozen blastocyst transfer - Authors' reply. Lancet 2019;394:1228.

6. Shi Y, Sun Y, Hao C, et al. Transfer of Fresh versus Frozen Embryos in Ovulatory Women. N Engl J Med 2018;378:126-36.

7. Shapiro BS, Daneshmand ST, Garner FC, et al. Evidence of impaired endometrial receptivity after ovarian stimulation for in vitro fertilization: a prospective randomized trial comparing fresh and frozen-thawed embryo transfers in high responders. Fertil Steril 2011;96:516-8.

8. Shapiro BS, Daneshmand ST, Garner FC, et al. Evidence of impaired endometrial receptivity after ovarian stimulation for in vitro fertilization: a prospective randomized trial comparing fresh and frozen-thawed embryo transfer in normal responders. Fertil Steril 2011;96:344-8.
9. Aflatoonian A, Oskouian H, Ahmadi S, et al. Can fresh embryo transfers be replaced by cryopreserved-thawed embryo transfers in assisted reproductive cycles? A randomized controlled trial. J Assist Reprod Genet 2010;27:357-63.

10. He Y, Zheng H, Du H, et al. Delayed frozen embryo transfer failed to improve live birth rate and neonatal outcomes in patients requiring whole embryo freezing. Reprod Biol Endocrinol 2020;18:1.

11. Bourdon M, Santulli P, Maignien C, et al. The interval between oocyte retrieval and frozen-thawed blastocyst transfer does not affect the live birth rate and obstetrical outcomes. PLoS One 2018;13:e0206067.

12. Ozgur K, Bulut H, Berkkanoglu M, et al. Frozen embryo transfer can be performed in the cycle immediately following the freeze-all cycle. J Assist Reprod Genet 2018;35:135-42.

13. Lattes $\mathrm{K}$, Checa MA, Vassena $\mathrm{R}$, et al. There is no evidence that the time from egg retrieval to embryo transfer affects live birth rates in a freeze-all strategy. Hum Reprod 2017;32:368-74.

14. Santos-Ribeiro S, Polyzos NP, Lan VT, et al. The effect of an immediate frozen embryo transfer following a freeze-all protocol: a retrospective analysis from two centres. Hum Reprod 2016;31:2541-8.

15. Li H SX, Yang J, Li L, Chen J, Lu X, Zhang W, Ng E. Comparison of the ongoing pregnancy rate of immediate versus delayed frozen-thawed embryo transfer following a stimulated IVF cycle: a prospective randomized controlled trial. Hum Reprod 2019;34:i29-i30.

16. Higgins C, Healey M, Jatkar S, et al. Interval between IVF stimulation cycle and frozen embryo transfer: Is there a benefit to a delay between cycles? Aust N Z J Obstet Gynaecol 2018;58:217-21.

17. Kaye L, Marsidi A, Rai P, et al. Frozen blastocyst transfer outcomes in immediate versus delayed subsequent cycles following GnRH agonist or hCG triggers. J Assist Reprod Genet 2018;35:669-75.

18. Huang J, Lu X, Xie Q, et al. Timing of frozen-thawed embryo transfer after controlled ovarian stimulation in a non-elective freeze-all policy. Ann Transl Med 2019;7:752.

19. Pinborg A. IVF/ICSI twin pregnancies: risks and prevention. Hum Reprod Update 2005;11:575-93.

20. McLernon DJ, Harrild K, Bergh C, et al. Clinical effectiveness of elective single versus double embryo transfer: meta-analysis of individual patient data from randomised trials. BMJ 2010;341:c6945. 
21. Kolibianakis EM, Zikopoulos K, Smitz J, et al. Elevated progesterone at initiation of stimulation is associated with a lower ongoing pregnancy rate after IVF using $\mathrm{GnRH}$ antagonists. Hum Reprod 2004;19:1525-9.

22. Junovich G, Mayer Y, Azpiroz A, et al. Ovarian stimulation affects the levels of regulatory endometrial NK cells and angiogenic cytokine VEGF. Am J Reprod Immunol 2011;65:146-53.

Cite this article as: Drakopoulos P, Santos-Ribeiro S, Mackens S, Racca AL, Blockeel C, Tournaye H, Makrigiannakis A. To delay or not frozen embryo transfer in freeze-all cycles? Ann Transl Med 2020;8(13):812. doi: 10.21037/atm.2020.03.209
23. Conrad KP, Baker VL. Corpus luteal contribution to maternal pregnancy physiology and outcomes in assisted reproductive technologies. Am J Physiol Regul Integr Comp Physiol 2013;304:R69-72.

24. Horcajadas JA, Minguez P, Dopazo J, et al. Controlled ovarian stimulation induces a functional genomic delay of the endometrium with potential clinical implications. J Clin Endocrinol Metab 2008;93:4500-10. 